\title{
Quantitative intonation modeling of interrogative sentences for Mandarin Speech Synthesis
}

\author{
$\mathrm{Ya} \mathrm{Li}^{\mathrm{a}}$, Jianhua Tao ${ }^{\mathrm{a}, \mathrm{b}, \mathrm{c}}$, Wei Lai ${ }^{\mathrm{a}, \mathrm{d}}$ and Xiaoying $\mathrm{Xu}^{\mathrm{a}, \mathrm{d}}$ \\ ${ }^{a}$ National Laboratory of Pattern Recognition, Institute of Automation, \\ Chinese Academy of Sciences, Beijing, China \\ ${ }^{\mathrm{b}} \mathrm{CAS}$ Center for Excellence in Brain Science and Intelligence Technology, \\ ${ }^{\mathrm{c}}$ School of Computer and Control Engineering, \\ University of Chinese Academy of Sciences, Beijing, China \\ ${ }^{\mathrm{d}}$ Department of Chinese Language and Literature, Beijing Normal University, Beijing, China \\ \{yli,jhtao\}@nlpr.ia.ac.cn, laiwei_0508@126.com, xuxiaoying2000@bnu.edu.cn
}

\begin{abstract}
Previous intonational research on Mandarin has mainly focused on the prosody modeling of statements or the prosody analysis of interrogative sentences. To support related speech technologies, e.g., Text-to-Speech, the quantitative modeling of intonation of interrogative sentences with a large-scale corpus still deserves attention. This paper summarizes our work on the quantitative prosody modeling of interrogative sentence in Mandarin. A large-scale natural speech corpus was used in this study. By extracting the pitch contours and fitting the intonation curves, we found that $F_{0}$ declination and final lowering both existed in interrogative sentences, while they were claimed to be absent in Mandarin in some previous studies. In addition, the declination function could be modeled linearly, and the bearing unit of final lowering in Mandarin was found to be the last prosodic word in the utterance, regardless of its length, rather than a fixed duration range. It was argued in this study that the difference between this finding and the commonly believed rising intonation of the interrogative sentences resulted from the nonlinear relationship between prosody production and perception. The underlying mechanism for the existence of $F_{0}$ declination and final lowering in interrogative sentences is also discussed.
\end{abstract}

Index Terms- $\mathrm{F}_{0}$ declination; intonation; interrogative sentences; final lowering; prosody

\section{INTRODUCTION}

$\mathrm{B}$ Y contrast to the long history of work on incorporating intonation models into Text-to-Speech systems to generate natural $\mathrm{F}_{0}$ contours for synthesized English declarative and interrogative utterances (Mattingly, 1966; Qian et al., 2011; Santen and Moebius, 2000; Taylor, 2000), research on $F_{0}$ contour generation in Mandarin TTS systems has focused much more on syllable-level tone contrasts, including models of tonal coarticulation across syllables and interaction with phrase-level downtrends in declarative statements (Prom-on et al., 2009; Qian et al., 2011; Shih and Sproat, 1998; Yuan and Liberman, 2014).

Unlike the literature on English interrogative intonation patterns, research on Chinese interrogative intonation is complicated because of the conflict on pitch perception between tone and intonation (Ren et al., 2013). Moreover, much of the instrumental literature on $\mathrm{F}_{0}$ contours in Mandarin questions has focused on declarative questions -- i.e., questions where there is no morphosyntactic marking of the interrogative function. Previous instrumental studies model the differences between the intonational effects on declarative and interrogative utterances in terms of the following aspects. a) Trends of top/base lines; Gårding (1987) modeled Chinese intonation with grids, which qualitatively marks a time-varying pitch range that is different for interrogative intonation and declarative intonation. Shen adjusted Gårding's grid model and suggested a gentle fall on the top line and a slight rise on the base line for interrogative sentences (Gasrding, 1987; Shen, 1994). Lee (2005) found a rise on the top line and significant expansion of the pitch range are the crucial localized $F_{0}$ cues in yes-no questions. The expansion of pitch range was also found crucial in (Yang, 1995). b) Starting point: Shen (1990) proposed that all types of questions begin with a higher pitch register than statements. c) Boundary tone: Lin adopted autosegmental-metrical theory and emphasized the role of boundary tone in the distinction between interrogative and declarative intonation (Lin, 2004, 2006). d) Phrase curve and strength: Yuan suggested that an overall higher phrase curve and higher strength on final syllables accounts for interrogative intonation in Chinese (Yuan et al., 2002). For questions with question words or final particles, the interrogative intonation would have more variations (Wang, 2008).

Although considerable studies have been carried out on interrogative intonation in Mandarin, they cannot fully satisfy the needs of Text-to-Speech. For one thing, these studies focus on the general intonation trend of questions (Shen, 1994; Shen, 1990; Yuan et al., 2002), whereas more concrete details of pitch variation within the whole sentence are still eagerly needed to support Text-to-Speech. For instance, is pitch variation distributed evenly over the whole sentence, or is it mainly realized by specific parts of the sentence? Such issues are still under debate. For another thing, to generate natural prosody, conclusions from experimental corpus should be verified in large-scale natural speech. Since Chinese is a tonal language, 
previous studies on intonation tended to adopt designed stimuli to control the effect of tone (Shih, 2000; Wang et al., 2012; Xu, 2006; Xu and Wang, 1997; Yuan, 2004; Yuan et al., 2002); there are only a few studies in the literature about the interrogative intonation of Chinese in large-scale natural speech (Lee, 2005). To make up for these inadequacies, this paper particularly examined the global and utterance-final $\mathrm{F}_{0}$ trends in a corpus of recorded utterances of question-answer pairs selected from online transcriptions of interviews in the Chinese University of Communication Media Language Corpus, to investigate the detailed prosody variations, and thereby contributes to the generation of natural prosody in Text-to-Speech.

The next two subsections review the relevant previous literature on $\mathrm{F}_{0}$ declination and final lowering, which are the two constructs that have been proposed as relevant in differentiating statements from questions in many languages. Previous studies found a downtrend of the overall pitch through the utterance for many languages, especially in statements (Pierrehumbert, 1979). There are many factors that can lead to a pitch downtrend. One is the declination effect, which refers to the tendency of $\mathrm{F}_{0}$ to decline over the course of an utterance and which has been observed in many languages (Cohen et al., 1982; Ladd, 1984; Pierrehumbert, 1979; Shih, 2000; Umeda, 1980). Another is final lowering, referring to an additional lowering effect near the end of the sentence (Liberman and Pierrehumbert, 1984).

\section{1. $F_{0}$ declination}

$\mathrm{F}_{0}$ declination has been found in many languages and for a long time has been treated as a universal property of speech intonation. Many intonation models adopt declination as a baseline upon which more local $\mathrm{F}_{0}$ patterns reside.

The trend line of $\mathrm{F}_{0}$ declination has been modeled in different ways by previous studies, for example, explicit (Lieberman et al., 1985) and implicit modeling of $\mathrm{F}_{0}$ declination (Fujisaki, 1983). Explicit $F_{0}$ declination is described by fitting trend lines to the $\mathrm{F}_{0}$ contour of the utterance or to certain salience points in the contour. Some studies characterized declination with a single line, e.g., a linear regression line fitted to all $\mathrm{F}_{0}$ points (Lieberman et al., 1985). Others suggested that two separate reference lines are required to characterize $\mathrm{F}_{0}$ declination, i.e., the topline that connects the peak values of $\mathrm{F}_{0}$ and the baseline that connects the valley values, because the two lines show different patterns in coding linguistic structures (Cooper and Sorensen, 2012; Gårding, 1979; Maeda, 1976; O'Shaughnessy, 1976). By contrast, the implicit modeling of declination has been mostly obtained by mathematical modeling instead of empirical observation, for instance, in Fujisaki model (Fujisaki, 1983) accent command responses are superimposed onto the exponentially decaying phrase component, and the $\mathrm{F}_{0}$ contour is the sum of the responses from the phrase components and the accent components.

The above characteristics of declination reveal another dichotomy: whether declination is straight or bent. Fitting straight lines to $\mathrm{F}_{0}$ values facilitates the observation of the trend and the slope of declination and is widely used in intonational research, ( e.g., Hart and Collier, 1979; Maeda, 1976). However, researchers have found that the declining rate is not always consistent within the whole utterance. Cooper and Sorensen (2012) modeled the declination by two successive straight lines, the steeper one is prioritized, indicating that the declining rate is faster at the beginning and becomes slower approaching the end of the utterance. In both Pierrehumbert's model (Pierrehumbert, 1980) and Fujisaki's model, the declination line decays exponentially as a function of time. Yuan and Liberman (2014) compared the topline and baseline of Chinese and English, and found that in English, both topline and baseline show an initial plateau, a middle declination and a final lowering, while in Chinese Mandarin, the topline is similar to English and the baseline is close to a straight line.

The declination trend was found to be closely related to utterance duration in plenty of previous research. Briefly speaking, a longer utterance starts at a higher pitch and contains a shallower slope (Cooper and Sorensen, 2012; Gårding, 1979; Hart and Collier, 1979; Maeda, 1976; Swerts et al., 1996). This relationship is considered as a sign of the existence of phrase-scale preplanning. Thorsen (1980) reported that statements have the steepest falling contours; syntactically unmarked questions have the gentlest ones, and in between these two endpoints are other types of questions and non-terminal statements. When more factors such as inter-speaker variation are taken into consideration, the relationship between declination trend and utterance duration turned out to be unclear in some previous studies (Heuven, 2004; Laniran and Clements, 2003; Liberman and Pierrehumbert, 1984; Prieto et al., 2006; Prieto et al., 1996).

The physical mechanism of declination has been investigated as well and several mechanisms/principles have been put forward, e.g., a drop in subglottal air pressure (Collier, 1975; Gelfer et al., 1983; Lieberman, 1967), a "tracheal pull" which lowers the sternum and the larynx (Maeda, 1976), or a "laziness principle" (Vaissière, 1983). However, it is still under debate whether declination is totally passive or whether it somehow involves active control of the respiratory muscles to signal linguistic meaning. If declination also involves some kind of phonological process, then this phonological nature lies in the fact that declination can be used for normalization in speech perception. For example, listeners would perceive two peaks as equally high in pitch when the second is acoustically lower (Pierrehumbert, 1979; Tseng, 2006). Another argument for its linguistic nature is that declination is dependent on sentence type; particularly, it is often suppressed in interrogative sentences (Thorsen, 1980).

As has been mentioned above, most previous research on intonation modeling was based on statements and we still do not know whether these modeling methods are applicable to interrogative sentences. Since the major prosodic difference between statements and interrogative sentences is the intonation, an accurate intonation model of interrogative sentences is still pressing need to the related speech technologies. 


\subsection{Final lowering}

The second factor for $\mathrm{F}_{0}$ drift down is final lowering, which indicates an additional lowering near the end of the utterance and has been found in many languages, e.g., Greek (Arvaniti and Godjevac, 2003), Danish (Thorsen, 1984), Dutch (Gussenhoven and Rietveld, 1988), Yoruba (Connell and Ladd, 1990; Laniran, 1993), Spanish (Prieto et al., 1996), German (Truckenbrodt, 2004), Japanese (Pierrehumbert and Beckman, 1988; Poser, 1984), Kipare (Herman, 1996) and Chinese (Lai et al., 2014a).

Potential physiological triggers of final lowering were first discussed by Liberman and Pierrehumbert (1984). They thought that a drop in the subglottal pressure and relaxation of the laryngeal muscles were necessary articulatory correlates to final lowering. This claim was followed by Herman, Beckman and Honda (1996), who considered final lowering as one of the products of an overall "vocal effort" decrease towards the end of an utterance.

Meanwhile, explanations from the linguistic point of view were also continuously being pursuit in many different languages. Final lowering could be used to signal the discourse finality (Herman, 2000), and to encode focus as a specific means of post focus compression (Xu, 1999; Xu and Wang, 1997). The absent of final lowering is used to show incredulity (Herman, 1996) in Kipare.

There are various methods for detecting final lowering, such as predicting the value of final peaks by data modeling (Liberman and Pierrehumbert, 1984; Truckenbrodt, 2004), comparing final vs. penultimate peaks (Arvaniti and Godjevac, 2003; Lai et al., 2014a), comparing $\mathrm{F}_{0}$ drops between successive peaks (Grabe, 1998), comparing mean $\mathrm{F}_{0}$ between final vs. penultimate syllables (Herman, 1996), and comparing the pitch of the same component in final vs. non-final positions (Maekawa, 2010; Shih, 2000). Arvaniti (2007) compared the effect of the former three methods and suggested that pitch comparison between final and penultimate peaks is more sensitive among all three approaches and thus is more suitable for final lowering detection, so we adopt this approach in this article. Concerning the influencing scope of final lowering, different conclusions have been obtained based on different measurements for final lowering; for instance, the last three syllables (Herman, 1996) as opposed to the last accent phrase (Arvaniti, 2007). This disagreement indicates that more effort should be made to find the basic bearing unit and the scope of final lowering.

\subsection{Research Goals}

Pitch downtrends like declination and final lowering are commonly considered as natural features of statements; nevertheless, they are suggested to be absent or suppressed in interrogative sentences (Vaissière, 1983). Thus it is interesting to explore the interaction between pitch downtrends and the interrogative mood and how this interaction shapes the final realization of pitch contour. Besides, the variation of declination and final lowering in different types of questions still remains unclear. The main goal of this study is to contribute a prosody model of Mandarin interrogative sentences to related speech technologies.

What makes our research worthwhile is that we employ a large-scale natural dialogue corpus to investigate the overall pitch trend throughout the sentence as well as pitch variation within the sentence for different question types. The aim of this paper is to model the intonation of interrogative sentence in a quantitative way. To this end, we evaluate whether or to what extent declination and final lowering exist in interrogative sentences, and whether their existence and realization are influenced by question type and other specific phonological properties. In the exploration of this issue, we also try to answer the following questions: do declination and final lowering in interrogative sentences share the same principles or mechanism with statements? For example: does declination show different trendline slopes in declarative sentences and different kinds of questions? What is the scope of final lowering? More importantly, how can we quantify declination in a more exact way so that we can improve the fitness of intonation models?

This research is not only expected to give us a better understanding of declination and final lowering in the specific phonological situation - in interrogative sentences-it is also supposed to expand our previous knowledge on the prosody of interrogative sentences in the following ways. First, we use a large scale corpus to investigate the prosody of questions instead of a small scale one in most of the previous research. Second, since Chinese is a tonal language, the observed $\mathrm{F}_{0}$ contour is the result of the combination of many effects, e.g., intonation and tone. The factors in prosody modeling are teased apart in a large scale corpus, rather than ruling out tonal effects by designed corpus, e.g., making up a question with syllables of the same tone. Third, we provide a quantitative intonation model for interrogative sentence which further extends the previous research on the statistical analysis of the interrogative sentence.

The rest of the paper is organized as follows. Section 2 introduces the large scale corpus we used. Section 3 answers the question of whether $\mathrm{F}_{0}$ declination exists in interrogative sentences; in addition, it also makes exploration towards more accurate quantification of declination. Section 4 investigates whether final lowering exists in interrogative sentences. If true, what is the realization of final lowering in different phonological environments? Section 5 discusses the mechanism of $\mathrm{F}_{0}$ declination and final lowering in interrogative sentences. Section 6 concludes this paper.

\section{CORPUS}

This paper extends the previous lab speech work by using a large scale natural corpus to verify the findings obtained from the designed experimental corpora, which is an inevitable step toward applying the intonation model of questions to speech technologies.

\subsection{Question taxonomy}

Based on syntax structure and pragmatic function, questions in Chinese can be divided into six types: wh-question, v-neg-v 
questions, alternative questions, particle yes-no questions and tag questions.

An example of each type of question is listed as follows.

Wh-questions:

ni3-de5 lao3-shi1 shi4 shui2?

you-GEN teacher be who

Who is your teacher?

Alternative questions:

ni3 xi3-huan1 hong2-se4 hai2-shi4 lan2-se4?

you like red or blue

Do you like red or blue?

Particle yes-no questions:

ni3 leng3 ma5?

you cold PRT

Are you feeling cold?

Tag questions:

ni3 shuo1 ying1-yu3, shi4-ma5?

you speak English, yes PRT

You speak English, don't you?

V-neg-v questions:

ni3 kan4 bu2 kan4 dian4-ying3?

you watch not watch movie

Will you watch the movie (or not)?

Unmarked questions/declarative questions:

ni3 yao4 zou3?

you want leave

You are leaving?

Among these question types, the v-neg-v question offers two opposite possibilities to choose from in forming the answer. In Chinese, the $v$ in v-neg-v can be formed by a verb, an adjective, or an adverb. Unmarked questions/declarative questions (Cruttenden, 1997) have the same form with a statement, but have a question mark at the end of the sentence and the intonation of a question when uttered, which is why they are also called intonation questions (Lee, 2005). The wh- questions, particle yes-no questions and v-neg-v questions express the interrogative mood by lexical means. In more detail, the three types of questions convey doubt by wh- words, final neutral-tone particles "ba5" or "ma5", and the repetition of the predicate v-neg-v, respectively. Alternative questions and tag questions express the interrogative mood by particular syntactic structures or phrases.

Hereafter, this study uses the term questions in most cases to indicate interrogative sentences mentioned above for simplicity, although these two terms have slight difference (Lee, 2005).

\subsection{Natural corpus}

The large scale corpus chosen for this work is collected from Question and Answer Conversation Corpus (MLC) which contains more than 2000 pair of questions and answers. All the conversations are selected from Chinese interview programs and cover a wide range of topics. In the corpus construction, we firstly took the question mark "?" as the keyword to search in the whole database. However, not all the questions are followed by answers, and some of the question and answer pairs are not conduced for the sake of seeking/providing information. Therefore, not all the questions in the original corpus were selected in our final corpus. In some cases, the informal text, including regional vernacular was rewritten into a formal text. The whole procedure is a guided procedure, not just several steps of question-mark searches and collects. Because of the copyright issue we do not have access to the original audio data. These question and answer pairs were transliterated and then re-read by a trained male speaker in a professional recording studio in Chinese Academy of Science. In addition, in this work, we do not use multi-turn dialogs; only single turns each formed by one question and one answer is considered. For these two reasons, the speaker does not have any records of context when he uttered the sentence, and thus he does not have any context-specific attitude, intention, or emotion while uttering.

In the real dialog, a question could be uttered as an Information-Seeking Question or Echo-Question, which are two different utterance types (Lee, 2005) and may have different prosody patterns. For example, "what was that like?" could be uttered with a rising final boundary pitch movement if there is preceding context of the conversation, but when there is no other context, e.g., dialog history; people tend to use the default pitch pattern, i.e., the falling pitch to utter this sentence. In our corpus, there is no preceding and following context; the speaker did not have any conversation history or context when he uttered the question, so a default intonation is used. In addition, during the recording process, the speaker was asked to maintain a natural speaking style without acting or exaggeration. Based on the above analysis, it is safe to state that all the questions in our corpus are Information-Seeking Question in terms of the utterance type classification (Lee, 2005).

The reason for using speech data from one speaker is that the ultimate goal of our work is integrating the proposed intonation model of interrogative sentences into Text-to-Speech systems. In dealing with the multiple-speaker corpus in our previous work, we indeed found prosodic differences in aspects such as the location of focus, speech rate, and locations of phrase boundaries in different speakers. Admittedly, different speakers have different speaking styles and intonation characteristics, which all sound natural and correct, and should be discussed in prosody modeling. However, although we can use the speaker normalization method, e.g., the semitone method, it is not the best way to generate synthetic speech with consistent style and high quality.

The six types of interrogatives introduced in subsection 2.1 are included in this corpus, and the distribution of each type question type is listed in Table 1.

Four levels of prosodic units (syllable, prosodic word, prosodic phrase and intonation phrase) were annotated and manually checked by research assistants (Liu et al., 2008) for all the questions used in this study. Fig. 1 shows an example of the four levels of prosodic hierarchy, in which, PW, PP, IP represent prosodic word, prosodic phrase and intonation phrase, respectively. 
TABLE 1. DISTRIBUTION OF DIFFERENT INTERROGATIVES

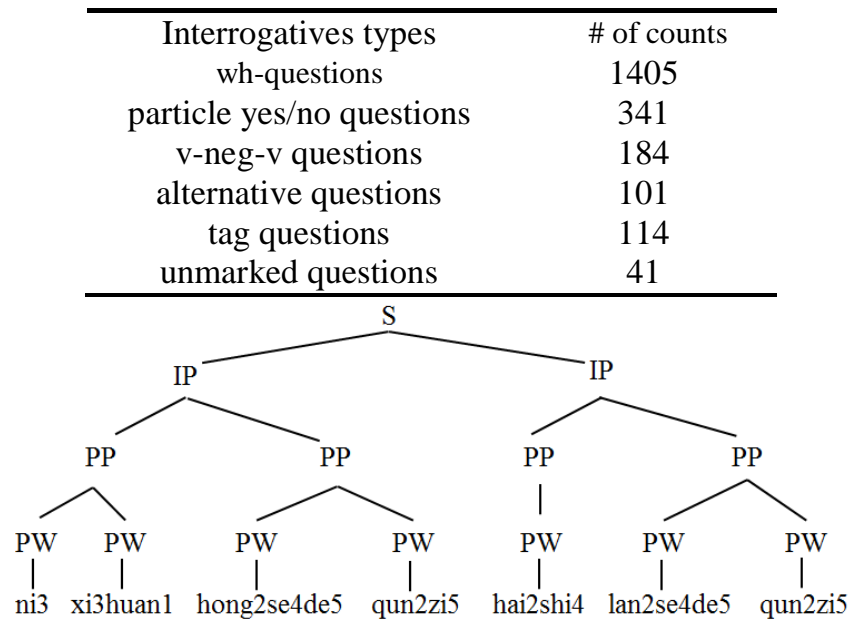

Figure 1: The prosodic hierarchy of the sentence "ni3 xi3-huan1 hong2-se4-de5 qun2-zi5 hai2-shi4 lan2-se4-de5 qun2-zi5? (Do you like red skirt or blue one?)"

\section{3. $\mathrm{F}_{0}$ DECLINATION}

\section{1. $\quad F_{0}$ modeling on natural corpus}

To verify that $\mathrm{F}_{0}$ declination exists in natural speech, we then carried out an $\mathrm{F}_{0}$ fitting experiment on the large scale corpus. Because what we aim to find is the intonation pattern, the longer sentence, which includes more than one intonation phrases, was segmented into several intonation phrases. The slope of the $\mathrm{F}_{0}$ regression line was extracted to measure the $\mathrm{F}_{0}$ trend. The pitch contours were automatically extracted by STRAIGHT (Kawahara et al., 1999) on these intonation phrases, and basic smoothing and cubic spline interpolation (Mixdorff et al., 2003) were performed to remove outliers. Cubic spline interpolation is very a fundamental numerical analysis method and implemented in many open-source toolkits, and performed by Matlab in our work. The starting and ending silence of the utterance were removed to get rid of their effect in intonation fitting; otherwise, the spline interpolation does not work well. A low pass Butterworth filter of 5-order with a stop frequency of $0.5 \mathrm{~Hz}$ was used to extract the low frequency contour (LFC), inspired by the Fujisaki model (Mixdorff et al., 2003). Although we have different objectives with (Mixdorff et al., 2003), his idea of LFC extracting could be employed in our work, since our work aims to propose a quantitative model of intonation of questions which could be used in Text-to-Speech systems to improve the expressiveness of the synthetic speech. Then linear $\mathrm{F}_{0}$ fitting was carried out to obtain the intonation trend. This procedure is shown in Fig. 2.

We used Eq. (1) to fit the pitch contours. The slope of the fitted lines corresponds to $a$ in Eq. (1), which represents the slope of the intonation trend.

$$
F_{0}=a x+b
$$

In our corpus,

$a=-0.016 \pm 0.013$, and

$b=141.68 \pm 46.54$,

which indicates that the average declination rate is $16 \mathrm{~Hz} / \mathrm{s}$. In Shih's work (2000), the declination rate of statements in Mandarin were $34 \mathrm{~Hz} / \mathrm{s}, 25 \mathrm{~Hz} / \mathrm{s}, 18 \mathrm{~Hz} / \mathrm{s}$ and $17 \mathrm{~Hz} / \mathrm{s}$ for their four speakers. Fig. 3 shows the results of each step in intonation trend extraction from natural $\mathrm{F}_{0}$ contour.

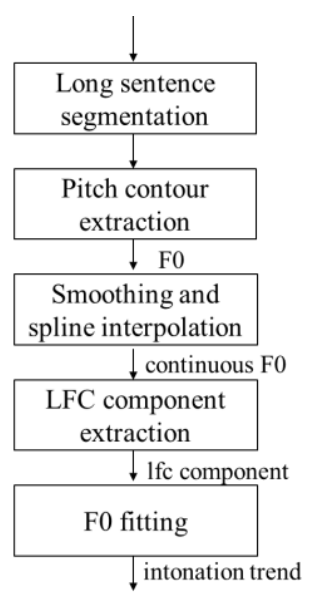

Figure 2: Flowchart of intonation trend extraction.

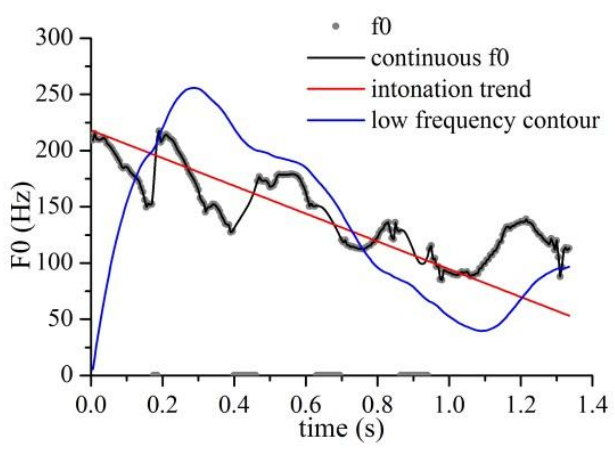

Figure 3: Intonation trend extraction from natural $\mathrm{F}_{0}$ contour.

Figure 4 shows the distribution of linear fitting coefficients: $a$ and $b$. The mean and variation of $a$ are -0.016 and 0.013 respectively. For comparison, the intonation contours from the questions and statements are both shown in Fig. 4. It can be seen that most of the intonation slopes are negative in both statements and questions. In addition, the slope in question intonation has a more compact distribution than that in statements. This phenomenon will be discussed in Section 5.

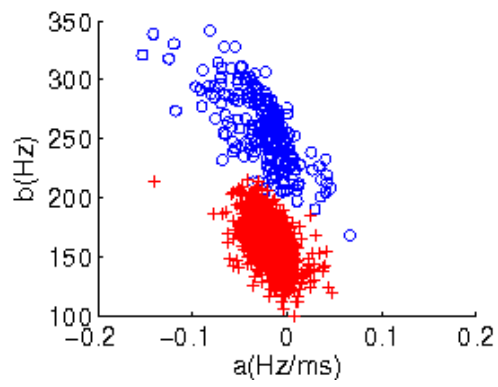

Figure 4: Linear fitting coefficients distribution of the intonation contours. (red crosses, questions; blue circles, statements)

Since the utterance slope and the starting pitch of the intonation contours correlate with utterance duration, which was verified by the previous research, we then further demonstrate the relationship between $a, b$ and duration of natural corpus in Fig. 5 and Fig. 6. Similar trends are found, the 
intonation slope and starting pitch have a positive correlation with the duration, which can be seen from the trend line in Fig. 5 and Fig. 6. The detailed $F_{0}$ declination of each question type will be illustrated in Section 4.

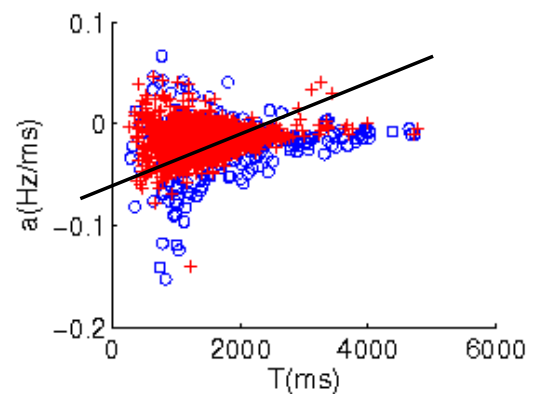

Figure 5: Distribution of intonation slope $a$ along duration T (red crosses, questions; blue circles, statements; black line, trend line.)

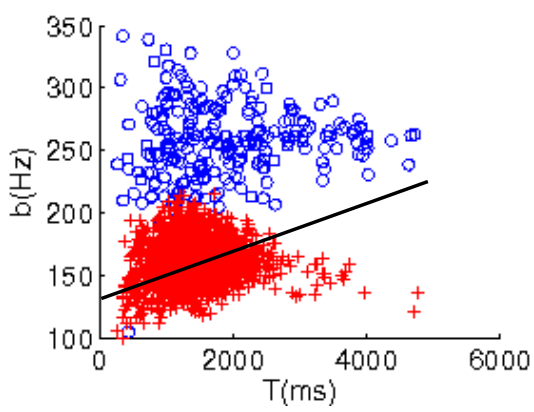

Figure 6: Distribution of the starting pitch along duration $\mathrm{T}$ (red crosses, questions; blue circles, statements; black line, trend line.)

\section{FINAL LOWERING}

This section studies the $F_{0}$ variations near the end of the sentence. We will determine whether final lowering exists in questions. If yes, what is the bearing unit of final lowering, e.g., syllable or word, and how can we model it?

\subsection{Existence of final lowering}

\subsubsection{ANOVA of the final $F_{0}$ variations}

This experiment studied the $\mathrm{F}_{0}$ variations at the final portion of the sentence, with particular concerns on the prosodic phenomenon of the final lowering effect.

For each utterance we extracted the following parameters from raw $\mathrm{F}_{0}$ contour:

- $\quad$ Peaks and valleys of the last five prosodic words; and

- Syllable count of the last prosodic word.

We excluded those samples that:

- $\quad$ were made of less than five prosodic words; and

- $\quad$ ended up with neutral tone syllables (except for particle yes-no questions)

With these data, statistical analysis was performed. We extracted the toplines and baselines of the last five prosodic words to investigate the intonation trend. The topline was obtained by fitting the local $\mathrm{F}_{0}$ peaks in an utterance, while the baseline was obtained by fitting the local $F_{0}$ valleys in an utterance (Lai et al., 2014a; Lai et al., 2014b; Yuan and
Liberman, 2014). The error bars in Fig. 7 directly describe the pitch variations of the last five prosodic words in different types of sentences. The sentence types we studied included v-neg-v questions, wh- questions, particle yes-no questions, unmarked questions and statements. The distribution of each type of sentence is listed in Section 2. Further analysis of variance (ANOVA) post hoc tests were performed to compare the differences in pitch between adjacent prosodic words. Hereafter, * stands for a significant difference ( Sig. < .05); ** stands for an extremely significant difference (Sig. <0.01); and n.s. stands for no significance.

As shown in Fig. 7, pitch falls on toplines are not evenly distributed over the whole sentence. An additional lowering effect appears at the end of all types of sentences except for unmarked questions, which is usually referred to as final lowering. Unlike toplines, baselines are encoded by a consistent pitch fall throughout the selected parts in most types of sentences. Therefore, the final lowering effect is supposed to be realized mainly by toplines.

Final lowering can be obviously detected in wh- questions, v-neg-v questions and statements. Pitch drops much more rapidly when it comes to the last prosodic word (around 30-40 $\mathrm{Hz}$ ) than the former ones (around $10 \mathrm{~Hz}$ ). Since sentences with neutral tone syllable endings were removed from the samples, the above result was not affected by the final neutral syllables.

In particle questions, unstressed final particles were not removed and possibly made a contribution to the final pitch drop. However, a rapid fall also takes place between the last $2^{\text {nd }}$ prosodic word and the last $3^{\text {rd }}$ one. Since there is no evidence suggesting that a neutral-tone syllable would affect the pitch of its preceding syllables, the penultimate pitch drop can reasonably be attributed to the final lowering effect.

The existence of final lowering in $v-n e g-v$ questions and particle questions is supported by the statistical results, since extremely significant differences were detected between the last (and last $2^{\text {nd }}$ for particle questions) pairs of prosodic words on the toplines. As for the wh- questions and statements, there were also significant differences in other positions. This is because pitch variations in natural speech are more severe unstable than that in tone-effect controlled speech. However, an extra pitch drop other than consistent pitch declination can still be easily observed for wh- questions and statements.

Figure 7 shows two types of baseline encodings. One is a consistent declination throughout the selected part of sentences, which occurs in wh-questions, v-neg-v questions and statements. The other is an additional final drop near the end of the sentence, which occurs in particle yes-no questions. In unmarked questions, the drop takes place in the last two prosodic words. Coincidentally, in particle yes-no questions, if the last neutral-tone particles are excluded, the extra drop also occurs on the last two prosodic words.

Statistical results on baselines make a good fit with pitch variations by error bars. The difference between each pair of adjacent prosodic words is significant in wh- questions, v-neg-v questions and statements, suggesting a general smooth fall on baselines. For unmarked questions and particle questions, the pitch differences stay non-significant except for the last two or 

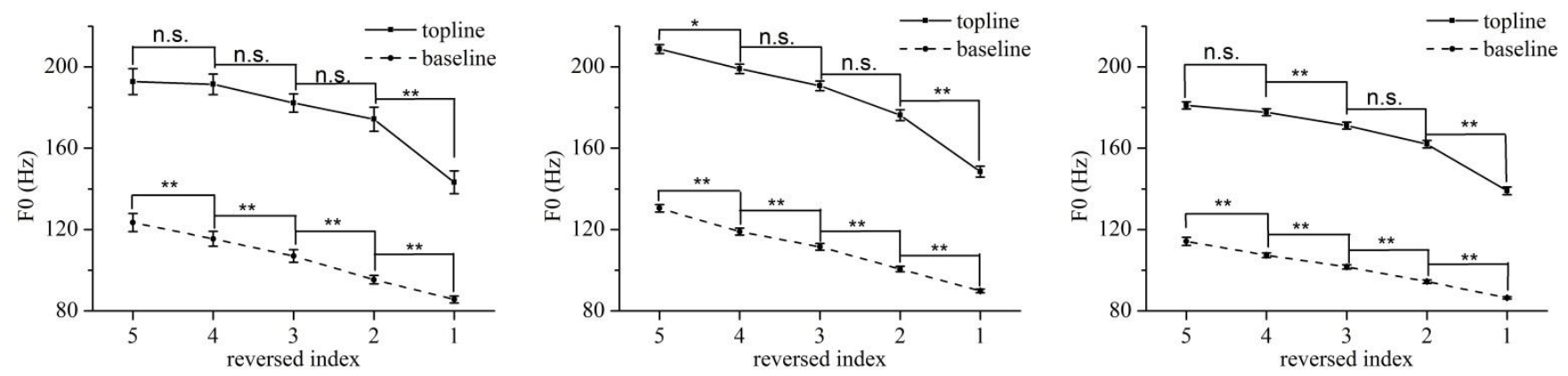

(a) Topline and baseline in v-neg-v questions (b) Topline and baseline in wh-questions (c) Topline and baseline in statements
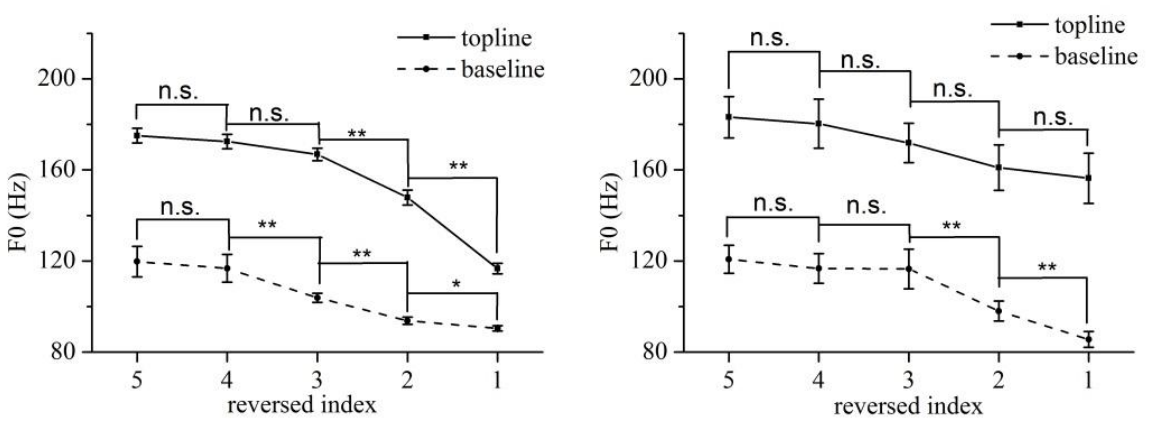

(d) Topline and baseline in particle yes-no questions (e) Topline and baseline in unmarked questions

Figure 7. Pitch variation of the last five prosodic words in five types of questions and statements.

three prosodic words. However, in general, the pitch falls on baselines are distributed more evenly than that on toplines.

\subsubsection{Elimination of tone effect}

Although an extra pitch drop near the end of the sentence is observed in Fig. 7, it has not yet been completely confirmed by statistical data. Thus, a supporting experiment is needed to eliminate tone differences and to narrow pitch gaps.

The complexity of tone combination in prosodic words introduces many problems into this research, because the pitch information in tone and intonation is hard to decompose. To make up for such disadvantages, a supporting experiment was conducted to control tone effects by comparing syllable-scaled pitch tone-by-tone. Since final lowering is mainly realized on topline according to previous results, only the maximal pitch of the last six syllables in each sentence was extracted and compared in this section.

Five pairs of syllables (last $1^{\text {st }}$ and last $2^{\text {nd }}-$ last $5^{\text {th }}$ and last $\left.6^{\text {th }}\right) \times 5$ tones $=25$ comparisons were performed. Based on ANOVA analysis, a pitch falling with a p-value which smaller than 0.05 is considered as significant lowering. Table 2 shows that very few significant pitch lowering were detected between the former three syllables (last 6 and 5, last 5 and 4). Only T2 syllables made a difference in wh- questions. Significant differences mainly occurred between the last three pairs of syllables, among which, the highest significance rate lies between the last $2^{\text {nd }}$ and $3^{\text {rd }}$ syllables.
To make this comparison clearer, the rate of significant lowering between each pair of adjacent syllables was calculated, as shown in Eq. (2).

$$
\text { lowering Rate }=\frac{\text { Numbers of Significant Lowerings }}{\text { Number of Comparisons }}
$$

TABLE 2. Syllable PAIRS WITH SignifiCANT Pitch DifFERENCES

\begin{tabular}{cccccc}
\hline syllable & Last & Last & Last & Last & Last \\
comparison & $6 \& 5$ & $5 \& 4$ & $4 \& 3$ & $3 \& 2$ & $2 \& 1$ \\
wh- & $\mathrm{T} 2$ & $\mathrm{~T} 2$ & $\mathrm{~T} 1, \mathrm{~T} 4$ & $\mathrm{~T} 1, \mathrm{~T} 2$ & $\mathrm{~T} 1, \mathrm{~T} 2$, \\
& & & & $\mathrm{T} 4, \mathrm{~T} 5$ & $\mathrm{~T} 4$ \\
statement & & & $\mathrm{T} 1, \mathrm{~T} 4$ & $\mathrm{~T} 1, \mathrm{~T} 2$ & $\mathrm{~T} 1, \mathrm{~T} 3$ \\
& & & $\mathrm{~T}, \mathrm{~T} 5$ & $\mathrm{~T} 4$, \\
v-neg-v & & & $\mathrm{T} 1, \mathrm{~T} 3$ & $\mathrm{~T} 2$ & $\mathrm{~T} 3$ \\
particle & & \multicolumn{3}{c}{ n.s. } \\
unmarked & & & \\
\hline
\end{tabular}

Table 3 shows the lowering rate between each pair of syllables calculated by Eq. (2). According to Table 3, a significant pitch lowering intensively occurs between the last three pairs of syllables, which approximately corresponds to the last prosodic word (usually, a prosodic word includes two to three syllables; one- and four- syllable prosodic words are rare). In wh- questions, rapid pitch fall exists throughout the whole sentence, but they are still most likely to appear between the last $2^{\text {nd }}$ pairs of syllables, with a lowering rate of 0.8 . The results verify that the existence of final lowering detected in this paper is independent of tone combinations. 
TABle 3. Lowering RATE Between EACH PAIR OF Syllables

\begin{tabular}{cccccc}
\hline Syllable & Last & Last & Last & Last & Last \\
Comparison & $6 \& 5$ & $5 \& 4$ & $4 \& 3$ & $3 \& 2$ & $2 \& 1$ \\
Wh- & 0.2 & 0.2 & 0.4 & 0.8 & 0.6 \\
Statement & - & - & 0.4 & 0.8 & 0.6 \\
V-neg-v & - & - & - & 0.4 & 0.2 \\
Particle & - & - & 0.4 & 0.2 & - \\
Unmarked & - & - & - & - & - \\
\hline
\end{tabular}

\subsubsection{Elimination of downstep effect}

Downstep and neutral tone effects (also considered as a kind of downstep in some work) are respectively triggered by low tone T3 and neutral tone T5. Downstep is most commonly described as the lowering effect caused by a low tone (L) on a following high tone $(\mathrm{H})$, such that a new, lower, "ceiling" is set for all subsequent Hs within a specific domain or prosodic unit. Since syllables of T3 and T5 mixed in the prosodic words may also result in pitch drops this experiment was designed to evaluate whether our result was influenced by downstep and neutral tone effects.

In this subsection, we excluded sentences with $\mathrm{T} 3$ or $\mathrm{T} 5$ on syllables preceding the lowering syllables and tested whether the lowering effect still exists. Wh- questions and statements were chosen to be studied in this subsection because of their large quantities (1405 and 2179 respectively).

The left error bar in Fig. 8 shows that final lowering in whquestions originally existed between the last $2^{\text {nd }}$ and $3^{\text {rd }}$ syllables. Then, sentences with their last $3^{\text {rd }}$ syllables carrying T3 or T5 were removed. Pitch variation of the qualified sentences is illustrated in the right panel of Fig. 8. It turns out that the only change is a pitch rise on the $3^{\text {rd }}$ to the last syllable, which is because of the exclusion of low tones. Nothing happened to the following two syllables. In this case, the abrupt lowering of the last $2^{\text {nd }}$ syllable is not caused by $\mathrm{T} 3$ or $\mathrm{T} 5$ of the last $3^{\text {rd }}$ syllables in wh- questions.
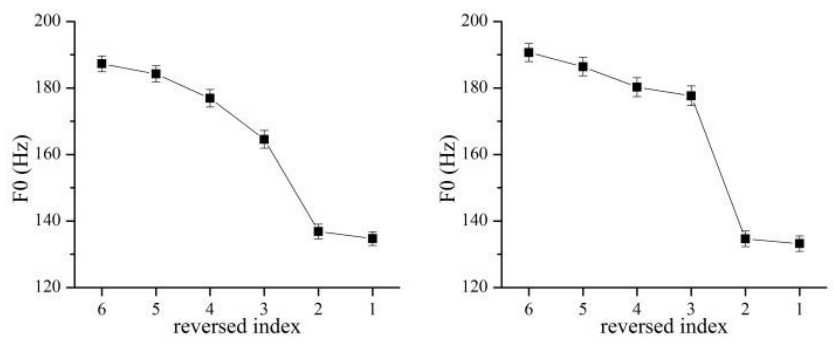

Figure 8. Original pitch fall (left) and after the exclusion of sentences with T3 and T5 (right) in wh- questions

In statements, a significant pitch fall originally occurs at the last $3^{\text {rd }}-1^{\text {st }}$ syllables, instead of the last $2^{\text {nd }}$ syllable in whquestions as shown in Fig. 9. Accordingly, the exclusion of sentences was performed with more syllables taken into account (the last $4^{\text {th }}-2^{\text {nd }}$ syllables). In consequence, a pitch rise appears on the last $4^{\text {th }}-2^{\text {nd }}$ syllables due to the exclusion of low tones. The extra pitch fall still occurs in the absence of $\mathrm{T} 3$ and T5. Based on the above analyses, we can deduce that our results are not affected by the downstep effect triggered by low tones.
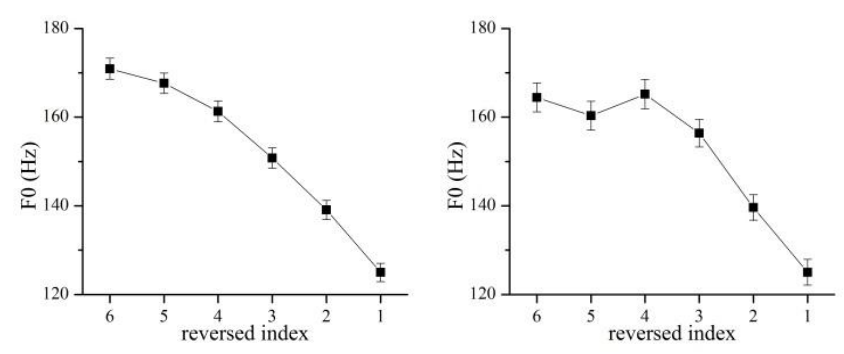

Figure 9. Original pitch fall (left) and after the exclusion of sentences with T3 and T5 (right) in statements

\subsection{Bearing unit of final lowering}

Since final lowering indeed exists in interrogative sentences, as verified by the above experiments, the next interesting question to ask is: what is the basic bearing unit of final lowering, syllable (SYL) or prosodic word (PW)? This question is important because it defines the modeling unit in quantitative models of intonation modeling. To ensure the credibility and generalizability of the result, this experiment employs different sentence types, namely, wh- questions, particle questions, v-neg-v questions and statements.

Sequential peaks of the last six PWs and the last six SYLs were extracted and plotted for different types of sentences, to investigate their final pitch variations. As shown in Fig. 10, the pitch contours on PW peaks are more similar among different types of sentences than those on SYL peaks. It can be observed that final lowering affects the last PW in all sentences intensively, with a final pitch fall $20-30 \mathrm{~Hz}$ larger than the former ones (except for particle questions whose sentence-final neutral-tone particles add another significant pitch fall to the end of the utterance). By contrast, pitch variation on SYL peaks shows more randomness. The greatest pitch lowering takes place between the last $2^{\text {nd }}$ and $3^{\text {rd }}$ SYL for wh- questions, and between the last $1^{\text {st }}$ and $2^{\text {nd }}$ and between $3^{\text {rd }}$ and $4^{\text {th }}$ SYL for v-neg-v questions and particle questions. For statements, the last three syllables are all affected and show even pitch falls.
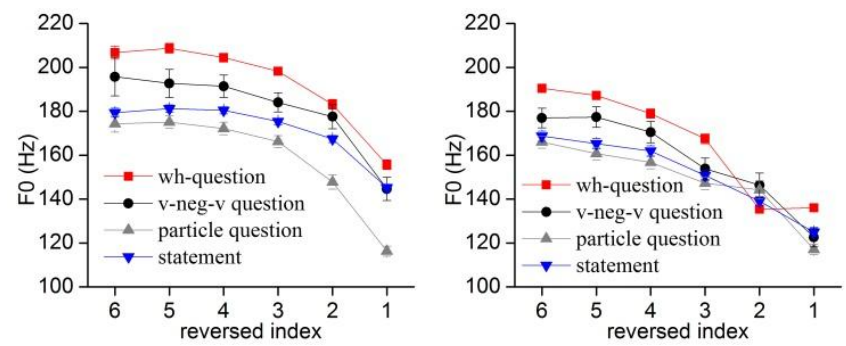

Figure 10. Peak variation on the last six PW (left) and that on the last six SYL (right) of wh- Q, particle Q, v-neg-v Q and statements

Further results from post hoc pairwise comparisons following the ANOVA test bring out similar conclusions. Extremely significant pitch differences (Sig. < 0.001) take place between the last pair of PW for wh- questions, v-neg-v questions and statements, and between the last two pairs of PW as well for particle questions (for the same reason mentioned above, final neutral tone particles). As for SYL comparison, extremely significant differences (Sig. $<0.001$ ) occur between 
the last $2^{\text {nd }}$ and $3^{\text {rd }}$, last $3^{\text {rd }}$ and $4^{\text {th }}$, and last $4^{\text {th }}$ and $5^{\text {th }}$ syllables for wh- questions, between the last $1^{\text {st }}$ and $2^{\text {nd }}$ and last $3^{\text {rd }}$ and $4^{\text {th }}$ syllables for $\mathrm{v}-\mathrm{neg}-\mathrm{v}$ questions and particle questions, and between the last $1^{\text {st }}$ and $2^{\text {nd }}$, last $2^{\text {nd }}$ and $3^{\text {rd }}$, and last $3^{\text {rd }}$ and $4^{\text {th }}$ syllables for statements.

Similar experiments have also been carried out on statements before (Lai et al., 2014a; Lai et al., 2014b), and we found that the bearing unit in statements is also PW. In the experiment on statements, the final prosodic words tend to undergo a rapid pitch fall of around $20 \mathrm{~Hz}$, regardless of their lengths, which indicates that final lowering is closely related to deeper phonological units rather than affecting a relatively fixed stretch from the end of the utterance.

\section{DISCUSSIONS}

With the experiments on a large-scale natural corpus, we found that $\mathrm{F}_{0}$ declination and final lowering do exist in interrogative sentences. This finding is slightly different from the previous research in that the rising intonation is claimed to be the characteristic of interrogative sentences.

Based on our quantified results on $\mathrm{F}_{0}$ declination in questions, we found that this contradiction is likely to be caused by two reasons. Firstly, we find that most of the questions have $F_{0}$ declination. However, Chinese questions have several types, and in some cases, variations and exceptions are found. Unmarked question may use a rising intonation, especially when they are short. Therefore, the observation that question uses rising intonation is impressionally true. Secondly, this contradiction could be caused by the nonlinear relationship between speech production and perception (Tuller et al., 2011) as we discussed in the introduction of this paper. In the perspective of speech production, there would be automatic by-products of respiratory activities, such as a drop in subglottal air pressure, decreased lung volume, and lowered sternum and larynx, which cause decreased vocal fold tension and $F_{0}$ fall. Ohala and Ewan (1973) suggested that it is more difficult to produce an $\mathrm{F}_{0}$ rise than a fall, because the laryngeal gesture requires more effort and takes more time, which is in line with the explanation of the "laziness principle". Therefore, to maintain or accelerate a rising pitch to convey doubt seems to be an effortful way to communication. Besides, if the sentence is long enough and its pitch needs to continue rising, it is easy to exceed an individual's pitch maximum. From the perspective of speech perception, previous studies have shown that listeners can perceive two peaks as equally high in pitch when the second is acoustically lower (Pierrehumbert, 1979; Tseng, 2006). Ohala (1978) argued that the tendency for $F_{0}$ downwards would lead listeners to expect such a decline and use it as a baseline in pitch perception. This constitutes one explanation that the phonological nature of declination lies in the fact that it is used for normalization in speech perception. Therefore, on one hand, the rationality of declination in questions agrees with speech production constraints. On the other hand, the listeners can expect the decline and compensate for it in perception, and this mechanism will enable listeners to detect interrogative mood when the $F_{0}$ contour is actually flat or slightly declines. The perceptual expectation is also used in the syntactic aspect of language comprehension (Levy, 2008), and prosodic pattern prediction (Brown et al., 2011).

The nonlinear relationship between speech production and speech perception caused confusion in the intonation modeling of speech. In the previous research, researchers tended to use a rising $\mathrm{F}_{0}$ contour to model interrogative utterances in speech synthesis. However, in this work, we have verified that the intonation of interrogative sentences actually declines in most cases. The declination by default and the lifting from the interrogative mood constitute the final slightly declined $\mathrm{F}_{0}$ contour of the interrogative sentence. Therefore, the downtrend of interrogative sentence is shallower than that of statements, which would result in a more compact distribution of declination rate. The superposition nature of the intonation of interrogative sentence could be used in speech technologies. For instance, in speech synthesis, superimposing a slight rising pitch contour onto the pitch contour generated from statements to finally construct an overall declining pitch contour would improve the naturalness of the synthetic speech.

\section{CONCLUSIONS}

This paper introduces our work on the quantitative intonation modeling of interrogative sentences. Different from previous research, a large-scale natural corpus is employed in this work to increase the credibility and generalizability of our work. The overall intonation trend of interrogative sentence is investigated and quantitatively modeled. We find that $\mathrm{F}_{0}$ declination also exists in interrogative sentences, including unmarked questions. The contradiction between our findings and generally believed rising pitch contour of interrogative sentences is argued to be caused by the nonlinear relationship between speech production and perception. In addition, our results support the existence of final lowering in both questions and statements, which experimentally answers the previous question of whether final lowering exists in Chinese. With regards to the bearing unit of final lowering, this research also finds that the basic bearing unit of final lowering in Chinese is the last prosodic word, regardless of its length and the sentence type, which lends support to the phonological nature of final lowering. The above conclusions would on one hand supply the previous prosodic structure theory with cross-linguistic evidence, and on the other hand contribute to prosody modeling and the generation of interrogative sentences of Chinese Mandarin in speech technology.

\section{ACKNOWLEDGMENTS}

This work is supported by the National High-Tech Research and Development Program of China (863 Program) (No. 2015AA016305), the National Natural Science Foundation of China (NSFC) ( No. 61305003, No. 61375027, No. 61425017, No. 61403386), the Major Program for the National Social Science Fund of China (13\&ZD189), and the Strategic Priority Research Program of the CAS (Grant XDB02080006).

\section{REFERENCES}

Arvaniti, A., 2007. On the presence of final lowering in British and American English. Tone and tunes. 2, 317-347. 
Arvaniti, A., Godjevac, S., 2003. The origins and scope of final lowering in English and Greek, Proceedings of the XVth International Congress of Phonetic Sciences, pp. 1077-1080.

Brown, M., Salverda, A.P., Dilley, L.C., Tanenhaus, M.K., 2011. Expectations from preceding prosody influence segmentation in online sentence processing. Psychonomic bulletin \& review. 18, 1189-1196.

Cohen, A., Collier, R., t HART, J., 1982. Declination: Construct or intrinsic feature of speech pitch? Phonetica. 39, 254-273.

Collier, R., 1975. Physiological correlates of intonation patterns. The Journal of the Acoustical Society of America. 58, 249-255.

Connell, B., Ladd, D.R., 1990. Aspects of pitch realisation in Yoruba. Phonology. 7, 1-29.

Cooper, W.E., Sorensen, J.M., 2012. Fundamental frequency in sentence production. Springer Science \& Business Media.

Cruttenden, A., 1997. Intonation. Cambridge University Press.

Fujisaki, H., 1983. Dynamic characteristics of voice fundamental frequency in speech and singing, The production of speech. Springer, pp. 39-55.

Gårding, E., 1979. Sentence intonation in Swedish. Phonetica. 36, 207-215.

Gasrding, E., 1987. Speech act and tonal pattern in Standard Chinese. Phonetica. 44, 13-29.

Gelfer, C.E., Harris, K.S., Collier, R., Baer, T., 1983. Is declination actively controlled. Vocal Fold Physiology. The Denver Center for the Performing Atrs, Inc., Denver, Colorado.

Grabe, E., 1998. Intonational phonology: English and German. Max-Planck Institute for Psycholoingusitics and University of Nijmegen.

Gussenhoven, C., Rietveld, A.C., 1988. Fundamental frequency declination in Dutch: testing three hypotheses. Journal of phonetics. 16, 355-369.

Hart, J., Collier, R., 1979. On the interaction of accentuation and intonation in Dutch, Proc. ICPhS pp. 395-402.

Herman, R., 1996. Final lowering in Kipare. Phonology. 13, 171-196.

Herman, R., 2000. Phonetic markers of global discourse structures in English. Journal of Phonetics. 28, 466-493.

Herman, R., Beckman, M., Honda, K., 1996. Subglottal pressure and final lowering in English, Fourth International Conference on Spoken Language, ICSLP 96. IEEE, pp. 145-148.

Heuven, V.J.v., 2004. Planning in speech melody: production and perception of downstep in Dutch. LOT Occasional Series. 2, 83-93.

Kawahara, H., Masuda-Katsuse, I., De Cheveigne, A., 1999. Restructuring speech representations using a pitch-adaptive time-frequency smoothing and an instantaneous-frequency-based F0 extraction: Possible role of a repetitive structure in sounds. Speech communication. 27, 187-207.

Ladd, D.R., 1984. Declination: a review and some hypotheses. Phonology. 1, 53-74.

Lai, W., Li, Y., Che, H., Liu, S., Tao, J., Xu, X., 2014a. Final Lowering Effect in Questions and Statements of Chinese Mandarin Based on a Large-scale Natural Dialogue Corpus Analysis, Speech Prosody, pp. 653-657.

Lai, W., Xu, X., Li, Y., Che, H., Liu, S., Tao, J., 2014b. Phonological influences on the realization of final lowering evidence from dialogue Chinese Mandarin, 2014 17th Oriental Chapter of the International Committee for the Co-ordination and Standardization of Speech Databases and Assessment Techniques (COCOSDA). IEEE, pp. 1-6.

Laniran, Y.O.., 1993. Intonation in tone languages: the phonetic implementation of tones in Yoruba. Cornell University, Department of Modern Languages and Linguistics.

Laniran, Y.O., Clements, G.N., 2003. Downstep and high raising: interacting factors in Yoruba tone production. Journal of Phonetics. 31, 203-250.

Lee, O.J., 2005. The prosody of questions in Beijing Mandarin. The Ohio State University.

Levy, R., 2008. Expectation-based syntactic comprehension. Cognition. 106, 1126-1177.

Liberman, M., Pierrehumbert, J., 1984. Intonational invariance under changes in pitch range and length, in: Aronoff, M., Oehrle, R.T. (Eds.), Language, Sound, Structure: Studies in Phonology Presented to Morris Halle by His Teacher and Students. MIT Press.

Lieberman, P., 1967. Intonation, perception, and language. MIT Research Monograph.

Lieberman, P., Katz, W., Jongman, A., Zimmerman, R., Miller, M., 1985. Measures of the sentence intonation of read and spontaneous speech in American English. The Journal of the Acoustical Society of America. 77, 649-657.

Lin, M., 2004. On production and perception of boundary tone in chinese intonation, the International Symposium on Tonal Aspects of Languages With Emphasis on Tone Languages, Beijing, China, pp. 125-130.

Lin, M., 2006. Yiwen he chenshu yuqi yu bianjiediao [Interrogative and Declarative Mood and Boundary Tone]. Zhongguoyuwen. 4, 364-376.
Liu, F., Jia, H., Tao, J., 2008. A Maximum Entropy Based Hierarchical Model for Automatic Prosodic Boundary Labeling in Mandarin, Chinese Spoken Language Processing, ISCSLP2008, China, pp. 257-260.

Maeda, S., 1976. A characterization of American English intonation. Massachusetts Institute of Technology.

Maekawa, K., 2010. Final lowering and boundary pitch movements in spontaneous Japanese, DiSS-LPSS, pp. 47-50.

Mattingly, I.G., 1966. Synthesis by rule of prosodic features. Language and Speech. 9, 1-13.

Mixdorff, H., Fujisaki, H., Chen, G.P., Hu, Y., 2003. Towards the automatic extraction of Fujisaki model parameters for Mandarin, Eighth European Conference on Speech Communication and Technology.

MLC, National Language Resources Monitoring and Research Center.

O'Shaughnessy, D.D., 1976. Modelling fundamental frequency, and its relationship to syntax, semantics, and phonetics. Massachusetts Institute of Technology.

Ohala, J., 1978. Production of tone, in: Fromkin, V. (Ed.), Tone: a linguistic survey. Academic Press, New York, pp. 5-39.

Ohala, J.J., Ewan, W.G., 1973. Speed of Pitch Change. Journal of The Acoustical Society of America. 53

Pierrehumbert, J., 1979. The perception of fundamental frequency declination. The Journal of the Acoustical Society of America. 66, 363-369.

Pierrehumbert, J., Beckman, M., 1988. Japanese tone structure. Linguistic Inquiry Monographs, 1-282.

Pierrehumbert, J.B., 1980. The phonology and phonetics of English intonation. Massachusetts Institute of Technology.

Poser, W.J., 1984. The phonetics and phonology of tone and intonation in Japanese. Massachusetts Institute of Technology.

Prieto, P., D'Imperio, M., Elordieta, G., Frota, S., Vigário, M., 2006. Evidence for'soft'preplanning in tonal production: Initial scaling in Romance, Speech Prosody, pp. 803-806.

Prieto, P., Shih, C., Nibert, H., 1996. Pitch downtrend in Spanish. Journal of Phonetics. 24, 445-473

Prom-on, S., Xu, Y., Thipakorn, B., 2009. Modeling tone and intonation in Mandarin and English as a process of target approximation. Journal of the Acoustical Society of America. 125, 405-424.

Qian, Y., Wu, Z.Z., Gao, B.Y., Soong, F.K., 2011. Improved Prosody Generation by Maximizing Joint Probability of State and Longer Units. IEEE Transactions on Audio Speech and Language Processing. 19, 1702-1710.

Ren, G.-Q., Tang, Y.-Y., Li, X.-Q., Sui, X., 2013. Pre-Attentive Processing of Mandarin Tone and Intonation: Evidence from Event-Related Potentials, in: Signorelli, F., Chirchiglia, D. (Eds.), Functional Brain Mapping and the Endeavor to Understand the Working Brain.

Santen, J.v., Moebius, B., 2000. A quantitative model of F0 generation and alignment, in: Botinis, A. (Ed.), Intonation Analysis Modeling \& Technology. Springer, pp. 269-288.

Shen, J., 1994. Intonation structure and intonation types of Chinese. Fangyan. 3, 221-228

Shen, X.-n.S., 1990. the prosody of Mandarin Chinese. Univ of California Press.

Shih, C., 2000. A declination model of Mandarin Chinese, Intonation: Analysis, Modelling and Technology. Springer, pp. 243-268.

Shih, C., Sproat, R., 1998. Issues in Text-to-Speech Conversion for Mandarin. Computational Linguistics and Chinese Language Processing. 1, 37-86.

Swerts, M., Strangert, E., Heldner, M., 1996. F0 declination in read-aloud and spontaneous speech, Fourth International Conference on Spoken Language, ICSLP 96. IEEE, pp. 1501-1504.

Taylor, P., 2000. Analysis and synthesis of intonation using the tilt model. Journal of Acoustic Society of America. 107, 1697-1714.

Thorsen, N., 1984. Intonation and text in Standard Danish. Annual Report Institute of Copenhagen. 18, 185-242.

Thorsen, N.G., 1980. A study of the perception of sentence intonation-Evidence from Danish. The Journal of the Acoustical Society of America. 67, 1014-1030.

Truckenbrodt, H., 2004. Final lowering in non-final position. Journal of Phonetics. 32, 313-348.

Tseng, C.Y., 2006. Higher level organization and discourse prosody, The Second International Symposium on Tonal Aspects of Languages, pp. 23-34.

Tuller, B., Nguyen, N., Lancia, L., Vallabha, G.K., 2011. Nonlinear dynamics in speech perception, Nonlinear Dynamics in Human Behavior. Springer, pp. 135-150.

Umeda, N., 1980. F0 Declination is situation dependent. The Journal of the Acoustical Society of America. 68, S70-S70.

Vaissière, J., 1983. Language-independent prosodic features, Prosody: Models and measurements. Springer, pp. 53-66. 
Wang, P., Shi, L., Shi, F., 2012. Declination and Downstep Effect in Declarative Sentences of Chinese Mandarin. Zhongguoyuyinxuebao. 3, 54-60. Wang, Y., 2008. Phonetic representation of interrogative tone in Chinese Mandarin, The 8th Phonetics Conference of China and the International Symposium on Phonetic Frontiers, Beijing, China.

$\mathrm{Xu}, \mathrm{Y} ., 1999$. Effects of tone and focus on the formation and alignment of $\mathrm{F} 0$ contours. Journal of phonetics. 27, 55-105.

$\mathrm{Xu}, \mathrm{Y}$., 2006. Principles of tone research, Proceedings of International Symposium on tonal aspects of languages, pp. 3-13.

$\mathrm{Xu}$, Y., Wang, Q.E., 1997. What can tone studies tell us about intonation?, Intonation: Theory, Models and Applications, pp. 337-340.

Yang, L.M., 1995. Sanzhong yiwenju de yudiao zhi yitong [Intonational Comparison of Three Types of Interrogative]. Zhongguo Yuwen, 144-152.

Yuan, J., 2004. Perception of Mandarin intonation, 2004 International Symposium on Chinese Spoken Language Processing. IEEE, pp. 45-48.

Yuan, J., C. Shih, Kochanski, G.P., 2002. Comparison of declarative and interrogative intonation in chinese, Speech Prosody, Aix-en-Provence, France.

Yuan, J., Liberman, M., 2014. F0 declination in English and Mandarin Broadcast News Speech. Speech Communication. 65, 67-74.

\section{APPENDIX}

In this appendix, some sample questions from large-scale Question and Answer Conversation Corpus are listed.

A.1 Abbreviation List:

PFV-perfective

PST-past

ACC-accusative

PRT-particle

CL-classifier

PROG-progressive

GEN-genitive

A.2 Questions:

Wh-questions:

1. zhe4 xue2-xiao4 zai4 na3-er5?

PRON school locate where

Where is this school?

2. shui2-shi4 di4-qiu2-shang4 zui4 ke3-ai4-de5 ren2?

who be earth on most lovely GEN person

Who is the cutest person on earth?

3. na4-ge5 shi2-hou4 jia1-li3-de0 sheng1-huo2 lai2-yuan2 shi4 shen2-me5?

what

That-CL time family-GEN livelihood source is

What is the family's source of livelihood at that time?

4. ge4-zhong3-ge4-yang4-de5 mo2-shu4 ni3 dou1 hui4 duo1-shao3?

Every kind every sort GEN magic you totally know how many

Among all kinds of magic, how many do you totally know?

5. shen2-me5 yuan2-yin1 rang4 ni3 yi4-zhi2 jian1-chi2-zhe5?

What reason make you always persevere PROG

What reason makes you always persevering?

6. ni3 dou1 zen3-me5 liao3-jie3 ta1-de5 xi2-xing4?

You totally how understand its habits

How do you totally understand its habits?

7. wan3 hui2-qu4 ji3-tian1 hui4 zen3-me5-yang4?

Late return several days will how

What will happen if (you) return several days later?

Alternative questions:

1. shang4-wang3 yi1-ban1 shi4 huo4-qu3 zhi1-shi2 hai2-shi4 wan2 you2-xi4?

Surfing usually acquire knowledge or play games
Does surfing usually (used to) acquire knowledge or play games?

2. ta1 hui4 xuan3-ze2 yi4-ping2 shui3 hai2-shi4 yi4-ping2 yin3-liao4?

He will choose one CL water or one CL drink?

He will choose a bottle of water or a bottle of drink?

3. ni3 zhe4 biao3-qing2 shi4 ku1 shi4 xiao4?

Your this looking be cry be laugh

Is the looking on your face crying or laughing?

4. ta1 shi4 nian2-ling2 tai4 xiao3 ne5 hai2-shi4 xin1-zhi4 bu4 cheng2-shu2 ne5? PRT

$\mathrm{He}$ be age too young PRT or mind not mature

Is he too young in age or immature in mind?

5. ni3-de5 shou3-ji1 shi4 nuo4-ji1-ya4-de5 hai2-shi4 san1-xing1-de5?

You-GEN cellphone be Nokia GEN or Samsung GEN

Is your cellphone Nokia or Samsung?

6. hua4-de5 shi4 yi4-zhi1 ya1-zi5 hai2-shi4 e2?

Draw GEN this one CL duck or goose

The drew one is a duck or a goose?

7. na4-li3-de5 kong1-qi4 shi4 hao3 shi4 huai4?

There GEN air be good be bad

Is the air there good or bad?

Particle yes-no questions:

1. ni3 gen1 ren2 da3-guo4-jia4 ma5?

You with people fight-PFV PRT

Have you fought with people?

2. gan3-jue2 jia1-xiang1 bian4-hua4 da4 ma5?

Feel hometown change big PRT

Do you feel the change of the hometown is big?

3. ni3 gang1-cai2 shuo1-de5 shi4 zhong3-zi5 ma5?

You just now talk GEN be seed PRT

Is it seed that you were talking about just now?

4. zhuan4 hen3-duo1 qian2 bu2 zhong4-yao4 ma5?

Earn much money not important PRT

Isn't earning much money important?

5. zhen1-de5 mei2 jue2-de2 ku3 ma5?

Really not feel bitter PRT

(You) really did not feel bitter?

6. ni3 yi3-qian2 zhong4-guo5 di4 ma5?

You before cultivate PFV land PRT

Have you cultivated land before?

7. neng2 gao4-su4 wo3 ni3 zai4 ji4-dian4 shui2 ma5?

Can tell me you PROG mourn for PRT

Can you tell me whom you are mourning for?

Tag questions:

1. ni3 xiang3-guo4 zhuan3-hang2, shi4-ma5?

You consider-PFV change job, yes PRT

You have considered changing a job, haven't you?

2. yun1-dao3-guo4 shi4-bu2-shi4?

Pass-out-PFV be-not-be

You have passed out, haven't you?

3. zhe4-ge4 qin2 hen3 zhong4-yao4 shi4-ba5?

This-CL piano very important yes-PRT

This piano is very important, isn't it?

4. tai2-wan1 ye3 qu4-guo5 shi4-bu2-shi4?

Taiwan also be PFV be-no-be

(You) have also been in Taiwan, haven't you?

5. na4-ge5 e4-yu2-qiu1 zui4 gui4 dui4-bu2-dui4?

That CL Alligator turtle most expensive yes-no-yes

That Alligator turtle is the most expensive, isn't it? 
6. shi2-san1 sui4 jiu4 chuo4 xue2 le5 shi4-ma5?

Thirteen years old drop of school PST yes PRT

You dropped out of school at thirteen years old, didn't you?

7. shi4 ni3 ti2-de5 jian4-yi4 shi4-ma5?

Be you give GEN suggestion yes PRT

It is you who give the suggestion, isn't it?

V-neg-v questions:

1. ta1 jiu1-jing4 lai2-bu2-lai2?

he actually come-not-come

Does he actually come or not?

2. shi4-bu2-shi4 hai2-yao4 bao3-chi2 yan2-su4 a5?

Be-not-be still need keep serious

Do (you) still to keep serious (or not)?

3. ni3 neng2-bu4-neng2 jiao1 wo3 yi4-zhong3?

You can-not-can teach me one kind

Can you teach me one kind (or not)?

4. you3-mei2-you3 jiao1 nv3-hai1-zi5 da3-de5 quan2?

Have-not-have teach girls do-GEN shadowboxing

Do you have shadowboxing that can be taught to girls?

5. na4 ren2-jia5 yuan4-yi4 bu2 yuan4-yi4?

Then they willing-not-willing

Then are they willing (or not)?

6. shi4-bu2-shi4 hen3 nan2?

Be-not-be very difficult

Is it very difficult (or not)?

7. shou4-bu2-shou4-dao4 gao1-yuan2 fan3-ying4-de5

ying2-xiang3?

undergo-not-undergo plateau effect GEN influence

Is it influenced by plateau effect?

Unmarked/declarative questions:

1. ta1-men5 ti2-chu1-le5 zhe4-ge4 yao1-qiu2?

They raise PST this request

They raised this request?

2. ye3-wai4 sheng1-cun2 ni3 mei2-you3 dao1-zi5?

Outdoor survival you not have knife

You don't have a knife during outdoor survival?

3. hou2-zi5 neng2 ren4-shi5 ni3-de5 sheng1-yin1?

Monkey can recognize you GEN voice

Monkeys can recognize your voice?

4. dui4-yu2 jie2-yue1 geng1-di4 hai2-hui4 you3 hao3-chu4?

For save arable land still have benefits

Still have benefits for saving arable land?

5. nin2 dui4 zhe4-ge4 hang2-xian4 te4-bie2 gan3-xing4-qu4?

You to this CL route especially interested

You are especially interested in this route?

6. zhe4-ge5 zhu3-ti2-qu3 shi4 ni3 xie3 de5?

This theme song be you write GEN

This theme song is written by you?

7. ni3-de5 jiao3 bei4 qia3-zhu4 le5?

You Gen foot ACC stuck PST

Your foot got stuck? 